\title{
Anti-windup compensation for systems with sensor saturation: a study of architecture and structure
}

\author{
Matthew C. Turner $\stackrel{*}{\text { * Sophie Tarbouriech }}{ }^{\dagger}$ \\ mct6ele.ac.uk tarbourelaas.fr
}

September 9, 2008

\begin{abstract}
This paper proposes a linear dynamic anti-windup strategy for the alleviation of performance and stability problems in systems which are linear apart from saturating sensors. Unlike anti-windup compensation for systems subject to actuator saturation, there is no agreed architecture for applying anti-windup to systems with sensor saturation and therefore attention is devoted to the discussion of various candidate configurations which can be interpreted as a particular choice of a static nonlinear map. The main results of the paper give existence conditions for compensators which yield global exponential stability and finite $\mathscr{L}_{2}$ gain of the overall nonlinear closed-loop system. The existence conditions are different to those which have appeared hitherto in the literature.
\end{abstract}

Keywords: Anti-windup, $\mathscr{L}_{2}$ gain, sensor saturation.

\section{Introduction}

The stability and performance problems associated with actuator saturation are well known to control engineers and have been responsible for a broad, sustained study in this area by the research community. Over the last twenty years, numerous results have been obtained, stemming from the fundamental theory established by [4, $21,15,6,17]$ and other authors, to the recent anti-windup based design techniques of $[16,28,3,20,25,8,7,9$, $30,29]$. Although much research remains to be completed, the engineer now at least has tools to enable him/her to cope - to some extent - with actuator saturation. The references [27] and [23] contain good selections of state-of-the-art research articles.

The study of systems in which saturation of the sensor is the chief concern is less developed, with only a few papers devoted to this topic $[22,14,11,13,2,10]$. This frugal treatment in the literature is perhaps due to the less frequent occurrence of sensor saturation, although, as it too introduces a nonlinearity into largely linear control loops, it is easy to see that it poses similar performance and stability issues. Sensor saturation is normally found in applications where cost prohibits the use of sensors with adequate range, leading to sensor saturation for large reference/disturbance inputs. Alternatively sensor saturation can model the situation where only the sign of the output is known. In this case, the sign function can be modelled by a saturation function with a steep gradient.

There are few prior results on systematic treatment of sensor saturation. Early work was pioneered by [11] where the observability properties of such systems were studied and [13] where a specific controller design strategy was presented. More recent work ([14]) suggests a low-gain control strategy. One interesting feature in [14] was that the existence of a (one-step) controller ensuring global stability of the (nonlinear) closed-loop

\footnotetext{
*Department of Engineering, University of Leicester, Leicester, LE1 7RH, UK

†Work supported by CNRS

${ }^{\dagger}$ LAAS-CNRS, University of Toulouse, 7 Avenue du Colonel Roche, 31077 Toulouse cedex 4, France
} 
system was ensured if the open-loop linear plant contained no invariant zeros in the open right half complex plane. This contrasts to the actuator saturation literature where a necessary and sufficient condition for the global stabilisation of systems with actuator saturation is that the open-loop plant must contain no poles in the open-right-half complex plane. However, it transpires that the condition on the invariant zeros given in [14], while sufficient for the control strategy advocated there, is not necessary in general. A further result appears in [2] where the Circle Criterion is used to design $\mathscr{H}_{\infty}$ controllers for linear systems with sensor nonlinearities. Finally, some work on sensor saturation also appeared in [10], where a dynamic saturated linear control law was proposed.

A naive appraisal of the sensor saturation problem suggests that it is similar to the actuator saturation problem with the plant and the controller interchanged. In fact this is not the case ([14, 22, 19]); one of the crucial differences between the two problems is the availability of the "un-saturated" signal. In the actuator saturation case, knowledge of both the output produced by the linear controller and the saturated version of this (i.e. the signals either side of the saturation block) is assumed. In the case of sensor saturation, it is not realistic to assume that the actual plant output is known; only the saturated version of this is known (otherwise there would be no problem!). This is problematic for the two-stage (anti-windup) approach taken here, and hence an observer is used to overcome this difficulty.

If the study of systems subject to sensor saturation is under-developed, the study of anti-windup compensation for this class of systems is less developed still. To the best of the authors' knowledge, the only literature discussing this approach are the papers $[19,22,26]$. The first of these papers considers so-called "state constrained" systems which can be interepreted as sensor-saturated systems when the plant direct feedthrough term is absent. [19] seems to be the first paper which proposes a systematic anti-windup design for the sensor saturation problem and the results given in that paper hint at some of the results given here. However, the work of [19] does not discuss the relative merits of different sensor saturation anti-windup architectures and only gives a single (albeit explicit) construction of an anti-windup compensator. Furthermore, the authors of this paper consider so-called total stability and, in essence, only prove that the closed-loop system trajectories are bounded, rather than exponentially stable. We shall discuss aspects of [19] as the paper progresses.

The latter two papers, [22] and [26] are the basis for the work presented here. These papers establish conditions for both local stability, and global stability and $\mathscr{L}_{2}$ gain, respectively, but use architectures which, as we shall show later, are probably not the best for the sensor saturation problem as they introduce another unnecessary nonlinearity into the system. Furthermore, in [26] a small oversight led to some slightly weaker results being reported.

The paper is organised as follows. The next section is devoted to the study of potential architectures for systems subject to sensor saturation. The subsequent section establishes necessary and sufficient conditions for the existence of a particular type of "anti-windup" compensator which yields global quadratic stability and finite $\mathscr{L}_{2}$ gain. Following this, a discussion of these conditions is given. Section 5 applies the synthesis conditions established in this paper to a simple example and finally conclusions are offered in Section 6.

Notation used in the paper is standard. The induced $\mathscr{L}_{2}$ norm, or finite $\mathscr{L}_{2}$ gain, of an operator $\mathscr{H}$ is denoted $\|\mathscr{H}\|_{i, 2}:=\sup _{0 \neq x \in \mathscr{L}_{2}} \frac{\|\mathscr{H} x\|_{2}}{\|x\|_{2}}$ where $\|x\|_{2}=\sqrt{\int_{0}^{\infty}\|x\|^{2} d t}$ is the $\mathscr{L}_{2}$ norm of the vector $x(t)$ and $\|x\|$ is its Euclidean norm. The $\mathscr{H}_{\infty}$ norm for a linear operator $P$ is defined as $\|P\|_{\infty}:=\sup _{\omega} \bar{\sigma}[P(j \omega)]$ where $\bar{\sigma}($.$) denotes the$ maximum singular value and $P(j \omega)$ is the frequency response matrix associated with the linear operator $P$. Equivalently, the $\mathscr{H}_{\infty}$ norm may be defined as $\|P\|_{\infty}=\|P\|_{i, 2}$. We denote the space of all positive definite symmetric $l \times l$ matrices as $\mathbb{R}_{+}^{l \times l}$ and the subspace of these which are diagonal as $\mathbb{D}_{+}^{l \times l}$. As normal, $I$ and 0 represent the identity and null matrices of appropriate dimensions, respectively. The orthogonal complement of a matrix $G \in \mathbb{R}^{n \times m}$ with $n>m$ and $\operatorname{rank}(G)=m$ is denoted $G^{\perp} \in \mathbb{R}^{(n-m) \times m}$ where the columns of $G^{\perp}$ span the null-space of $G^{\prime}$. 


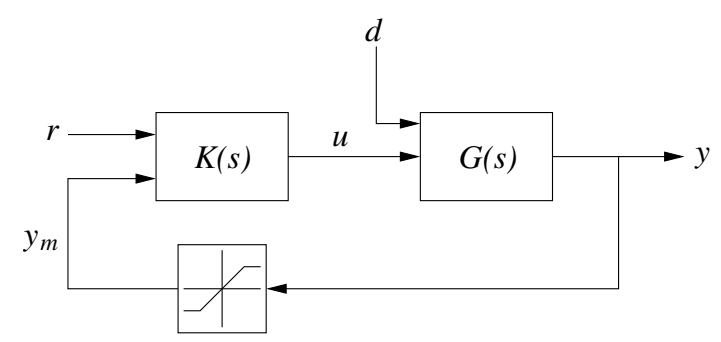

Figure 1: System with sensor saturation

\section{Anti-windup compensation for systems with sensor saturation}

\subsection{System description}

Consider Figure 1 where a system with sensor saturation is depicted. The plant $G(s)$ and the controller $K(s)$ are described by the following state-space equations

$$
\begin{aligned}
G(s) & \sim\left\{\begin{array}{l}
\dot{x}_{p}=A_{p} x_{p}+B_{p} u+B_{p d} d \\
y=C_{p} x_{p}
\end{array}\right. \\
K(s) & \sim \begin{cases}\dot{x}_{c} & =A_{c} x_{c}+B_{c} y_{m}+B_{c r} r \\
u & =C_{c} x_{c}+D_{c} y_{m}+D_{c r} r\end{cases}
\end{aligned}
$$

where $x_{p} \in \mathbb{R}^{n_{p}}$ is the plant state, $x_{c} \in \mathbb{R}^{n_{c}}$ is the controller state, $d \in \mathbb{R}^{n_{d}}$ is the disturbance, $r \in \mathbb{R}^{n_{r}}$ is the reference, $u \in \mathbb{R}^{m}$ is the manipulated plant input, $y \in \mathbb{R}^{q}$ is the output of the plant (used for feedback purposes) and $\operatorname{sat}(y)=: y_{m} \in \mathbb{R}^{q}$ is the output of the sensor.

To simplify the presentation, the plant is assumed strictly proper. The saturation and deadzone functions are defined as normal as

$$
\begin{gathered}
\operatorname{sat}(y)=y-\operatorname{Dz}(y) \\
\operatorname{sat}(y)=\left[\begin{array}{c}
\operatorname{sat}_{1}\left(y_{1}\right) \\
\vdots \\
\operatorname{sat}_{q}\left(y_{q}\right)
\end{array}\right], \quad \operatorname{Dz}(y)=\left[\begin{array}{c}
\mathrm{Dz}_{1}\left(y_{1}\right) \\
\vdots \\
\operatorname{Dz}_{q}\left(y_{q}\right)
\end{array}\right]
\end{gathered}
$$

where $\operatorname{sat}_{i}\left(y_{i}\right)=\operatorname{sign}\left(y_{i}\right) \min \left(\left|y_{i}\right|, \bar{y}_{i}\right) \forall i$ and $\operatorname{Dz}_{i}\left(y_{i}\right)=\operatorname{sign}\left(y_{i}\right) \max \left(0,\left|y_{i}\right|-\bar{y}_{i}\right) \forall i$. The positive scalars $\bar{y}_{i}>$ $0 \forall i \in\{1, \ldots, q\}$, represent the saturation levels. It is convenient to introduce the set

$$
\mathscr{Y}:=\left[-\bar{y}_{1}, \bar{y}_{1}\right] \times \ldots \times\left[-\bar{y}_{q}, \bar{y}_{q}\right]
$$

If the vector $y(t)$ resides within this set, we can expect linear behaviour, that is

$$
\operatorname{sat}(y)=y \quad \forall y \in \mathscr{Y}, \quad \operatorname{Dz}(y)=0 \quad \forall y \in \mathscr{Y},
$$

Assume first that $\operatorname{sat}(y) \equiv y$ (that is $\mathscr{Y}=\mathbb{R}^{q}$ ). Then, defining the interconnection of $G(s)$ and $K(s)$ through equations (1) and (7) as $[G, K]_{\text {nom }}$ (a state-space realisation of this is given in the appendix), i.e. the nominal linear closed-loop, we make the assumption

Assumption $1[G, K]_{\text {nom }}$ is asymptotically stable and well-posed.

As with the anti-windup literature on actuator saturation, we assume that $K(s)$ has been designed to ensure good behaviour in the absence of sensor saturation, and $K(s)$ may have been designed in ignorance of this saturation. 


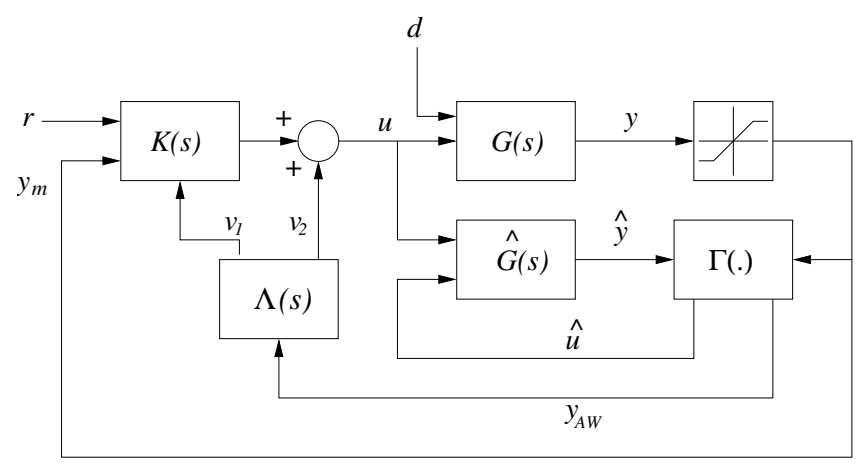

Figure 2: System with sensor saturation and "anti-windup" compensation

When $\operatorname{sat}(y) \neq y\left(\right.$ when $\mathscr{Y} \subset \mathbb{R}^{q}$ ), the interconnection depicted in the Figure 1 may exhibit undesirable performance and stability properties due to the nonlinearity present in the loop. To overcome the problem of sensor saturation, we would like to use an "anti-windup" like strategy where the system behaves nominally providing sensor saturation is not encountered, but, when sensor saturation is encountered, an additional compensator becomes active to limit performance degradation and to retain stability. Unlike actuator saturation where it is straightforward to implement this type of anti-windup strategy, the sensor saturation case is more problematic because we only know the saturated version of the output $y_{m}(t)=\operatorname{sat}(y(t))$ and do not know the actual "unsaturated" output, $y(t)$. Thus it appears difficult to duplicate the anti-windup strategy used in actuator saturation problems, for systems with sensor saturation problems. In this paper we explore some possible strategies which follow a similar philosophy.

Consider the system depicted in Figure 2, where again the nominal plant, with saturated sensor, and nominal controller are shown. The controller now has the state-space realisation

$$
K(s) \sim\left\{\begin{array}{l}
\dot{x}_{c}=A_{c} x_{c}+B_{c} y_{m}+B_{c r} r+v_{1} \\
u=C_{c} x_{c}+D_{c} y_{m}+D_{c r} r+v_{2}
\end{array}\right.
$$

where $v_{1} \in \mathbb{R}^{n_{c}}$ and $v_{2} \in \mathbb{R}^{m}$ are signals produced by the anti-windup compensator; during linear operation these signals are identically zero. The anti-windup compensator, $\Lambda(s)$, which becomes active when the signal $y_{A W} \neq 0$, is also shown. When $y_{A W} \neq 0$, the signals $v_{1}$ and $v_{2}$ become non-zero and, if $\Lambda(s)$ is designed appropriately, should enable stability and performance to be retained despite sensor saturation. Accompanying the anti-windup compensator is an observer, $\hat{G}(s)$ which is tasked with estimating the unsaturated output $y(t)$ as closely as possible. The observer is driven by the nominal control signal, $u(t)$, as normal, and also an "output injection" $\hat{u} \in \mathbb{R}^{q}$. Both the anti-windup compensator, $\Lambda(s)$ and the observer, $\hat{G}(s)$ are driven by the static nonlinearity $\Gamma: \mathbb{R}^{q} \times \mathbb{R}^{q} \mapsto \mathbb{R}^{q} \times \mathbb{R}^{q}$ which is defined as

$$
\left[\begin{array}{c}
\hat{u} \\
y_{A W}
\end{array}\right]=\Gamma\left(\left[\begin{array}{c}
\operatorname{sat}(y) \\
\hat{y}
\end{array}\right]\right)
$$

The map $\Gamma($.$) is central to the architecture of the sensor saturation anti-windup problem. Roughly speaking,$ the role of $\Gamma($.$) is to generate suitable signals \hat{u}$ (for driving the observer) and $y_{A W}$ (for driving the anti-windup compensator) from the saturated output $y_{m}=\operatorname{sat}(y)$ and the estimated output $\hat{y}$. It is essential to have some other means of generating $y_{A W}$ as $y$ itself is not available. The goal of $\Gamma($.$) therefore is to generate a suitable signal y_{A W}$ such that $y_{A W}$ is reasonably close to $y-\operatorname{sat}(y)$, so that the AW compensator is only active when saturation of the $\operatorname{sensors}$ occur $\left(y_{A W} \approx y-\operatorname{sat}(y)\right)$.

The architecture in Figure 2 is a generalisation of that used in [22] and [26]. The presence of the observer is crucial as we do not know the "unsaturated" output, $y$, and instead have to drive the anti-windup compensator with a signal, $y_{A W}$ generated from an estimate, $\hat{y}$ and $y_{m}=\operatorname{sat}(y)$. However, the signals $y_{A W}$ and $\hat{u}$ can be 


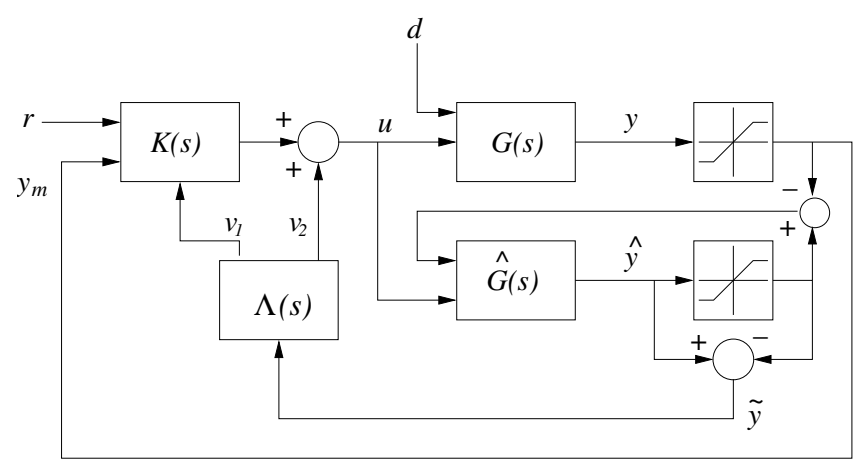

Figure 3: System with sensor saturation and "anti-windup" compensation

generated in different ways, effectively leading to different architectures for the sensor saturation problem. In the next subsection we discuss various candidates for $\Gamma$ and comment on their suitability.

Similar to $[12,8]$, we assign our anti-windup compensator the state-space realisation

$$
\Lambda(s) \sim\left\{\begin{array}{c}
\dot{x}_{a w}=\Lambda_{1} x_{a w}+\Lambda_{2} y_{A W} \\
{\left[\begin{array}{l}
v_{1} \\
v_{2}
\end{array}\right]=\left[\begin{array}{l}
\Lambda_{31} \\
\Lambda_{32}
\end{array}\right] x_{a w}+\left[\begin{array}{l}
\Lambda_{41} \\
\Lambda_{42}
\end{array}\right] y_{A W}}
\end{array}\right.
$$

and the observer has state-space equations

$$
\hat{G}(s) \sim\left\{\begin{array}{l}
\dot{\hat{x}}=A_{p} \hat{x}+B_{p} u+L \hat{u} \\
\hat{y}=C_{p} \hat{x}
\end{array}\right.
$$

We make the following standard assumption to ensure the existence of an appropriate observer gain, $L$.

Assumption 2 The pair $\left(C_{p}, A_{p}\right)$ is detectable. Equivalently there exists a matrix $L$ such that $A_{p}+L C_{p}$ is Hurwitz.

The goal of this paper is to use an appropriate static nonlinear map $\Gamma($.$) to establish conditions for the existence$ of an anti-windup compensator $\Lambda(s)$ to ensure that the nonlinear closed-loop behaves in some desirable way. The performance output is defined as

$$
z=C_{p z} x_{p}=: C_{2, \text { nom }} x_{\text {nom }}
$$

where $C_{2, \text { nom }}$ is defined in the appendix and $x_{\text {nom }}=\left[\begin{array}{ll}x_{p}^{\prime} & x_{c}^{\prime}\end{array}\right]^{\prime}$. The focus of the paper is then to consider the following problem.

Problem 1 Given a nonlinear static operator $\Gamma($.$) , find existence conditions for a compensator \Lambda(s)$, defined by equation (9) such that

1. The closed-loop system in Figure 2 (equivalently, that defined by equations (1), (7), (8), (9) and (10)) is globally quadratically (and thus exponentially) stable when $w=0$.

2. The map from $w$ to $z$ is finite $\mathscr{L}_{2}$ gain stable with gain $\gamma>0$. 


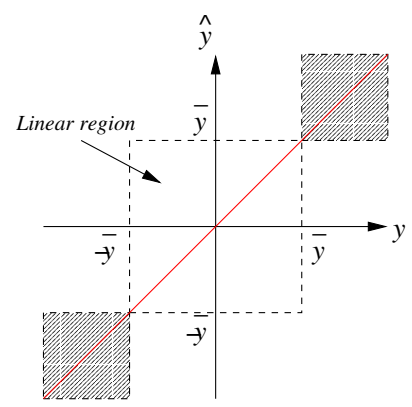

Figure 4: Region for which $\hat{u}=0$ - architecture 1

\subsection{Architecture 1: two nonlinearities, non-zero measure}

Perhaps the most intuitive architecture for the sensor saturation problem was proposed in [22] in which the antiwindup compensator is driven by the difference between an estimate of the output and a saturated version of this, and the observer is driven by the difference between the saturated output and the estimated saturated output. This is equivalent to $\Gamma($.$) being defined as$

$$
\left[\begin{array}{c}
\hat{u} \\
y_{A W}
\end{array}\right]=\Gamma\left(\left[\begin{array}{c}
\operatorname{sat}(y) \\
\hat{y}
\end{array}\right]\right)=\left[\begin{array}{c}
\operatorname{sat}(\hat{y})-\operatorname{sat}(y) \\
\hat{y}-\operatorname{sat}(\hat{y})
\end{array}\right]
$$

Thus the observer is driven by the difference between two saturated signals and the anti-windup compensator is driven by the "deadzoned" version of the estimated output, $\hat{y}$. This architecture was used in [22] in order to obtain local results and also in [26] for establishing global existence conditions. The architecture is shown explicitly in Figure 3. While this architecture can be seen as intuitive, there are some philosophical issues with its use. In particular it is of interest to discover the conditions under which the output injection vector $\hat{u}=0$. In linear system observers, this would correspond to the case when there is perfect reproduction of the estimated output, i.e. $\hat{y}=y$. However for our case, we can see that $\hat{u}=0$ under non-unique conditions, namely when $\operatorname{sat}(y)=\operatorname{sat}(\hat{y})$, which occurs when

$$
\left.\begin{array}{ll}
\text { (i) } & \hat{y}_{i} \geq \bar{y}_{i}, y_{i} \geq \bar{y}_{i} \\
\text { (ii) } & \hat{y}_{i} \leq-\bar{y}_{i}, y_{i} \leq-\bar{y}_{i} \\
\text { (iii) } \hat{y}_{i}=y_{i}
\end{array}\right\} \Rightarrow \hat{u}_{i}=0 \quad \forall i \in\{1,2, \ldots, q\}
$$

When $y$ and $\hat{y}$ are scalar, such conditions can be seen graphically and the hatched regions in Figure 4 show the value of $\left[\begin{array}{ll}\hat{y}^{\prime} & y^{\prime}\end{array}\right]^{\prime}$ for which $\hat{u}=0$, but $\hat{y} \neq y$. Note that unlike the linear case, the set

$$
\mathscr{Y}_{o b s, 1}=\left\{\left[\begin{array}{l}
y \\
\hat{y}
\end{array}\right] \in \mathbb{R}^{2 q}: \hat{u}=\operatorname{sat}(\hat{y})-\operatorname{sat}(y)=0\right\}
$$

does not have a measure of zero. Effectively this means that the output injection $\hat{u}$ is zero even though $\hat{y} \neq y$ and is relaying "false" information to the observer, $\hat{G}(s)$.

Another compelling reason for considering other architectures than that shown in Figure 3 is that there are more restrictive technical conditions on the existence of a globally stabilising anti-windup compensator: in [26] it was established that a necessary* condition was that the linear open-loop must be asymptotically stable. This is not possible when the linear controller contains integral modes, and thus such an architecture would be of less use in tracking problems. Indeed this was a major motivating factor in seeking alternative architectures.

\footnotetext{
*In that paper, they are also claimed to be sufficient, but this is, in fact, not the case.
} 


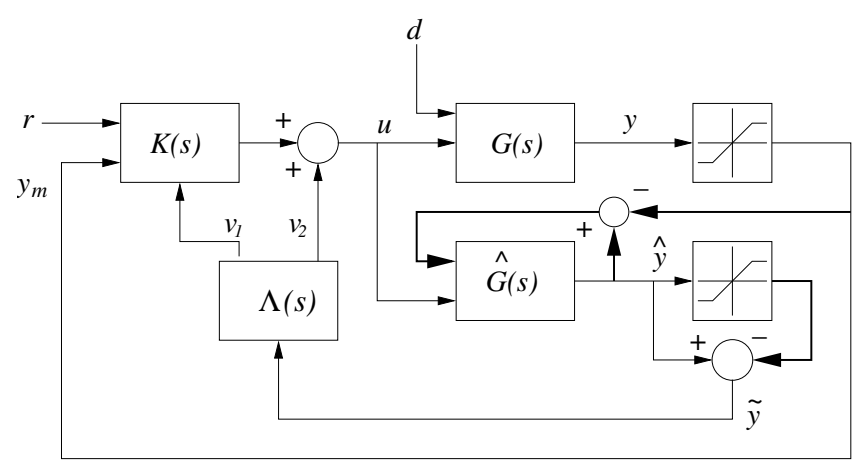

Figure 5: System with sensor saturation and "anti-windup" compensation

\subsection{Architecture 2: two nonlinearities, zero measure}

The philosophical problem of $\hat{u}=0$ when $\hat{y} \neq y$ can be remedied easily by driving the anti-windup compensator with the same signal as in architecture 1 , but by altering slightly the signal which drives the observer. Thus for this architecture, we choose $\Gamma($.$) as$

$$
\left[\begin{array}{c}
\hat{u} \\
y_{A W}
\end{array}\right]=\Gamma\left(\left[\begin{array}{c}
\operatorname{sat}(y) \\
\hat{y}
\end{array}\right]\right)=\left[\begin{array}{c}
\hat{y}-\operatorname{sat}(y) \\
\hat{y}-\operatorname{sat}(\hat{y})
\end{array}\right]
$$

The anti-windup architecture is shown explicitly in Figure 5. In this case, we find that $\hat{u}=0$ in the following cases

$$
\left.\begin{array}{l}
\text { (i) } \hat{y}_{i}=\bar{y}_{i}, y_{i} \geq \bar{y}_{i} \\
\text { (ii) } \hat{y}_{i}=-\bar{y}_{i}, y_{i} \leq-\bar{y}_{i} \\
\text { (iii) } \hat{y}_{i}=y_{i}
\end{array}\right\} \Rightarrow \hat{u}_{i}=0 \quad \forall i \in\{1,2, \ldots, q\}
$$

Thus, we see that output injection term, $\hat{u}$, is zero only when $y=\hat{y}$ or in the special case when $\hat{y}$ is exactly equal to its upper (lower) saturation limit and $y$ is operating in its upper (lower) saturation region. Again, assuming $\hat{y}$ and $y$ are scalars we can examine the set

$$
\mathscr{Y}_{o b s, 2}=\left\{\left[\begin{array}{l}
y \\
\hat{y}
\end{array}\right] \in \mathbb{R}^{2 q}: \hat{u}=\hat{y}-\operatorname{sat}(y)=0\right\}
$$

graphically and deduce that this set has measure zero - see Figure 6, where the dotted line marks the region where $\hat{u}=0$ and $\hat{y} \neq y$.

Remark 1: Although Architecture 2 seems a better choice from a philosophical perspective, it makes remarkably little difference to the anti-windup existence conditions whether Architecture 1 or 2 is chosen. Reference [26] uses the first architecture to derive conditions which ensure the existence of a compensator achieving global stability and finite $\mathscr{L}_{2}$ gain, but if a similar derivation to this is carried out using Architecture 2, the resulting existence conditions are essentially the same.

\subsection{Architecture 3: one nonlinearity, zero measure}

Architectures 1 and 2 are probably the most intuitive architectures for the sensor saturation problem as they follow exactly the same rationale as the actuator saturation problem: the anti-windup compensator is driven by 


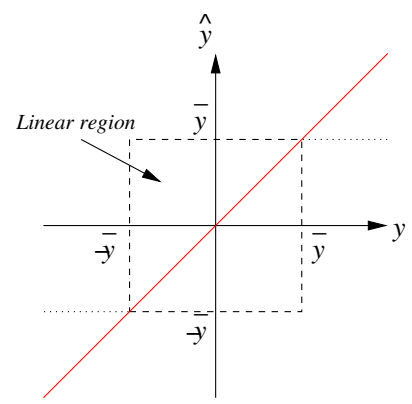

Figure 6: Region for which $\hat{u}=0$ - architecture 2

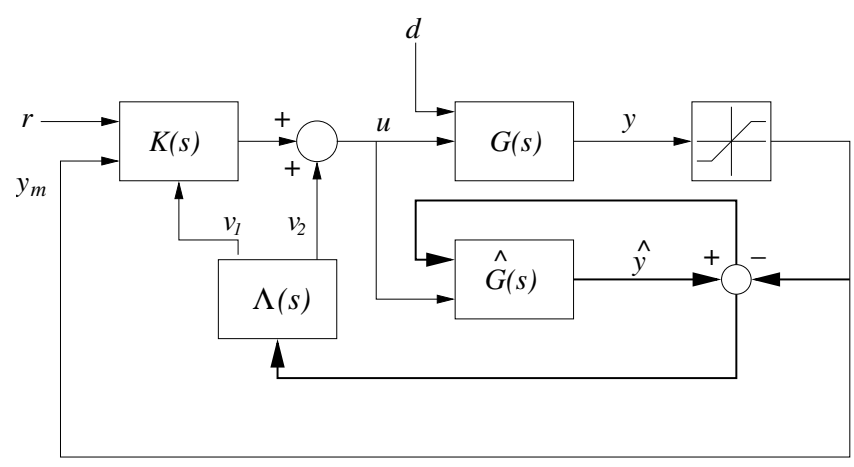

Figure 7: System with sensor saturation and "anti-windup" compensation

the difference between a signal and its saturated version, i.e. a "deadzoned" version of the signal. As $y$ itself is not available, this signal was chosen as $\hat{y}$ in the previous architectures, making $y_{A W}=\hat{y}-\operatorname{sat}(\hat{y})$. However, note that these architectures introduce an extra saturation nonlinearity into the problem in order to generate $\hat{y}$. It transpires that this extra nonlinearity makes existence conditions fairly complex (see [26]). Instead of introducing an extra nonlinearity, it appears more prudent to use the following signals to drive the observer and the anti-windup compensator. Thus $\Gamma($.$) is chosen as$

$$
\left[\begin{array}{c}
\hat{u} \\
y_{A W}
\end{array}\right]=\Gamma\left(\left[\begin{array}{c}
\operatorname{sat}(y) \\
\hat{y}
\end{array}\right]\right)=\left[\begin{array}{c}
\hat{y}-\operatorname{sat}(y) \\
\hat{y}-\operatorname{sat}(y)
\end{array}\right]
$$

Thus both observer and anti-windup compensator are driven by the difference between the estimated output and the saturated version of this. Note that only one nonlinearity, $\operatorname{sat}(y)$ appears in the architecture. This has the philosophical advantages of Architecture 2, discussed earlier, but it also prevents the addition of the extra saturation nonlinearity, sat $(\hat{y})$ which complicates the establishment of the stability and $\mathscr{L}_{2}$ gain conditions. This architecture is shown graphically in Figure 7.

Remark 2: In the anti-windup literature, it is customary to ensure that the anti-windup compensator is inactive unless actuator saturation occurs. This was formalised in [24] as requiring $v_{1}=0, v_{2}=0$ for all control signals which did not violate the saturation bounds, which can be interpreted as those signals not straying outside a certain set. In the sensor saturation case, this is equivalent to requiring

$$
y(t) \in \mathscr{Y} \forall t \geq 0 \Rightarrow v_{1}=0, v_{2}=0 \forall t \geq 0
$$

However, as our anti-windup compensator is driven by $y_{A W}$ which is generated by the static nonlinear map $\Gamma($.$) ,$ and we know that $y_{A W} \neq y-\operatorname{sat}(y)$, it may be the case that $y(t) \in \mathscr{Y}$ when $\hat{y}(t) \notin \mathscr{Y}$ (and vice versa) due to the 
error between the actual output and estimated output. Thus, our anti-windup compensator, $\Lambda(s)$, could be active unnecessarily or inactive when necessary. Assuming our observer is sufficiently fast and accurate, this is not thought to be a problem.

Remark 3: The anti-windup architecture used in [19] is almost the same as Architecture 3, with one additional assumption: $\hat{G}(s)$ has no observer gain $(L \equiv 0)$ making $\hat{G}(s)$ just a copy of $G(s)$. In addition to simplifying the problem, such a choice is attractive because, as will be seen later, it removes one of the matrix inequality constraints. The downside is that the "observer" output and the actual output could converge slowly.

\section{Main results}

The goal of this paper is to derive conditions which ensure existence of a globally exponentially stabilising antiwindup compensator which provides a finite $\mathscr{L}_{2}$ gain bound from exogenous input, $w$ to performance output, $z$. The existence conditions obtained are generally a function of the architecture of the AW compensator. In [26], the foregoing existence conditions were investigated using Architecture 1. For the reasons given in the previous section, concentration is hereafter focused on Architecture 3, in which only one nonlinearity (the physical sensor saturation) is present.

\subsection{State-space manipulations}

The main result is easier to state when some basic state-space manipulations have been carried out. As we consider Architecture 3 (Figure 7) we assume that $\hat{u}=y_{A W}=\hat{y}-\operatorname{sat}(y) \in \mathbb{R}^{q}$. For convenience we define the following vector valued signals:

$$
\begin{aligned}
\tilde{y} & =y-\operatorname{sat}(y) \in \mathbb{R}^{q} \\
\varepsilon & =\hat{x}_{p}-x_{p} \in \mathbb{R}^{n_{p}}
\end{aligned}
$$

Using these definitions it follows that

$$
\hat{y}-\operatorname{sat}(y)=\hat{y}-y+\tilde{y}=C_{p} \varepsilon+\tilde{y}
$$

where $\tilde{y}=\operatorname{Dz}(y)$. It then follows that a state-space realisation of the system (1), (7), (9) and (10) is

$$
\Sigma \sim\left\{\begin{array}{l}
\dot{\tilde{x}}=\tilde{A} \tilde{x}+\tilde{B}_{1} w+\tilde{B}_{2} \tilde{y} \\
y=\tilde{C}_{1} \tilde{x} \\
z=\tilde{C}_{2} \tilde{x}
\end{array}\right.
$$

where $\tilde{x}:=\left[\begin{array}{llll}x_{p}^{\prime} & x_{c}^{\prime} & x_{a w}^{\prime} & \varepsilon^{\prime}\end{array}\right]^{\prime} \in \mathbb{R}^{2 n_{p}+n_{c}+n_{a w}}$ represents the concatenated state of the system, $w=\left[\begin{array}{ll}r^{\prime} & d^{\prime}\end{array}\right]^{\prime} \in \mathbb{R}^{n_{w}}$ represents the exogenous inputs, and expressions for the tilded matrices can be found in the appendix. These matrices can be split to reveal a matrix of anti-windup compensator matrices (as in $[5,8]$ :

$$
\tilde{A}=A_{o}+\bar{B} \Lambda \bar{C} \quad \tilde{B}_{2}=B_{o}+\bar{B} \Lambda \bar{D}
$$

This decomposition is crucial to the development of the results and the matrices $A_{0}, B_{0}, \bar{B}, \bar{C}$ and $\bar{D}$ are described in the appendix.

\subsection{Preliminary results}

The main results are established using some well known results (see [1] and [5] for example). 


\section{Lemma 1 (Projection Lemma)}

The inequality $\Psi+G \Pi H^{\prime}+H \Pi^{\prime} G^{\prime}<0$ holds iff

$$
G^{\perp} \Psi\left(G^{\perp}\right)^{\prime}<0 \quad \text { and } \quad H^{\perp} \Psi\left(H^{\perp}\right)^{\prime}<0
$$

As we deal with the deadzone nonlinearity, $\tilde{y}=\mathrm{Dz}(y)$, we make use of the commonly used sector bound. This is known to be conservative, but allows tractable results to be obtained and also provides "robustness" to a class of sensor nonlinearities. In the remainder of the paper, we provide results assuming only that $\tilde{y}=\psi(y)$ and $\psi(.) \in \operatorname{Sector}[0, I]$. The central result we use is ([8]):

Lemma 2 (Quadratic stability and $\mathscr{L}_{2}$ gain) A state-space system

$$
\dot{x}=A x+B \psi(C x), \quad x \in \mathbb{R}^{n}, \psi: \mathbb{R}^{q} \mapsto \mathbb{R}^{q}
$$

is said to be quadratically stable with $\mathscr{L}_{2}$ gain $\gamma$ for all $\psi(.) \in \operatorname{Sector}[0, I]$ if and only if $\exists P \in \mathbb{R}_{+}^{n \times n}$ and $\exists W \in \mathbb{D}_{+}^{q \times q}$ such that the following inequality holds

$$
\dot{V}(x)+\gamma^{-1}\|z\|^{2}-\gamma\|w\|^{2}+2 \psi(u) W^{\prime}(u-\psi(u))<0
$$

for $V(x)=x^{\prime} P x>0$.

Finally, we shall need the following lemma from [18].

Lemma 3 Let $X, Y \in \mathbb{R}_{+}^{n \times n}$. Then the conditions

$$
\begin{aligned}
Y-X^{-1} & \geq 0 \\
\operatorname{rank}\left(Y-X^{-1}\right) & \leq n_{1}
\end{aligned}
$$

hold if and only if there exist matrices $Y_{12}, X_{12} \in \mathbb{R}^{n \times n_{1}}$ and $Y_{22}, X_{22} \in \mathbb{R}_{+}^{n_{1} \times n_{1}}$ such that

$$
\left[\begin{array}{cc}
X & X_{12} \\
X_{12}^{\prime} & X_{22}
\end{array}\right]^{-1}=\left[\begin{array}{cc}
Y & Y_{12} \\
Y_{12}^{\prime} & Y_{22}
\end{array}\right]>0
$$

\subsection{Existence conditions}

The main result gives a set of matrix inequalities which can be checked for the existence of a globally stabilising anti-windup compensator.

Theorem 1 Assume $\Gamma($.$) is given as in equations (8) and (18), then there exists an n_{a w}$ 'th order anti-windup compensator, $\Lambda(s)$, which solves Problem 1 with $\mathscr{L}_{2}$ gain $\gamma$ for all $\tilde{y}=\psi(y)$ where $\psi(.) \in \operatorname{Sector}[0, I]$ if and only if there exist matrices

$$
Q_{\text {nom }}=\left[\begin{array}{cc}
Q_{11} & Q_{12} \\
\star & Q_{22}
\end{array}\right] \in \mathbb{R}_{+}^{\left(n_{p}+n_{c}\right) \times\left(n_{p}+n_{c}\right)}, \quad R_{\text {nom }} \in \mathbb{R}_{+}^{\left(n_{p}+n_{c}\right) \times\left(n_{p}+n_{c}\right)}, \quad P_{44} \in \mathbb{R}_{+}^{n_{p} \times n_{p}} \quad W \in \mathbb{D}_{+}^{q \times q}
$$


such that the following matrix inequalities hold:

$$
\begin{aligned}
& {\left[\begin{array}{ccccc}
B_{p}^{\perp}\left(Q_{11} A_{p}^{\prime}+A_{p} Q_{11}\right)\left(B_{p}^{\perp}\right)^{\prime} & 0 & B_{p}^{\perp} Q_{11} C_{p}^{\prime} W & B_{p}^{\perp} B_{p d} & B_{p}^{\perp} Q_{11} C_{p z}^{\prime} \\
\star & \left(A_{p}+L C_{p}\right)^{\prime} P_{44}+P_{44}\left(A_{p}+L C_{p}\right) & P_{44} L & -P_{44} B_{p d} & 0 \\
\star & \star & -2 W & 0 & 0 \\
\star & \star & \star & -\gamma I & 0 \\
\star & \star & \star & \star & -\gamma I
\end{array}\right]<0} \\
& {\left[\begin{array}{cccc}
R_{\text {nom }} A_{\text {nom }}^{\prime}+A_{\text {nom }} R_{\text {nom }} & B_{0, \text { nom }}+R_{\text {nom }} C_{0, \text { nom }}^{\prime}(W) & B_{1, \text { nom }} & R_{\text {nom }} C_{2, \text { nom }}^{\prime} \\
\star & A_{p}^{\prime} P_{44}+P_{44} A_{p}-2 C_{p}^{\prime} W C_{p} & P_{44}\left[0-B_{p d}\right] & 0 \\
\star & \star & -\gamma I & 0 \\
\star & \star & \star & -\gamma I
\end{array}\right]<0} \\
& Q_{\text {nom }}-R_{\text {nom }} \geq 0 \\
& \operatorname{rank}\left(Q_{\text {nom }}-R_{\text {nom }}\right) \leq n_{\text {aw }}
\end{aligned}
$$

where expressions for the matrices $A_{\text {nom }}, B_{0, \text { nom }}, B_{1, \text { nom }}, C_{0, \text { nom }}, C_{2, \text { nom }}$ are given in the appendix.

Proof: If $\Gamma($.$) is given as in equations (8) and (18), the system under consideration has state-space realisation$ given by equation (23). From Lemma 2, we know this will be the case iff there exists $P \in \mathbb{R}_{+}^{\left(2 n_{p}+n_{c}+n_{a w}\right) \times\left(2 n_{p}+n_{c}+n_{a w}\right)}$, $W \in \mathbb{D}_{+}^{q \times q}$ such that

$$
\frac{d}{d t}\left(\tilde{x}^{\prime} P \tilde{x}\right)+2 \tilde{y}^{\prime} W\left(\tilde{C}_{1} \tilde{x}-\tilde{y}\right)+\gamma^{-1}\|z\|^{2}-\gamma\|w\|^{2}<0
$$

Substituting for $\tilde{x}$ and $z$ from equation (23) and applying the Schur complement, inequality (35) holds if and only if

$$
\left[\begin{array}{cccc}
\tilde{A}^{\prime} P+P \tilde{A} & P \tilde{B}_{2}+\tilde{C}_{1}^{\prime} W & P \tilde{B}_{1} & \tilde{C}_{2}^{\prime} \\
\star & -2 W & 0 & 0 \\
\star & \star & -\gamma I & 0 \\
\star & \star & \star & -\gamma I
\end{array}\right]<0
$$

which can be written as

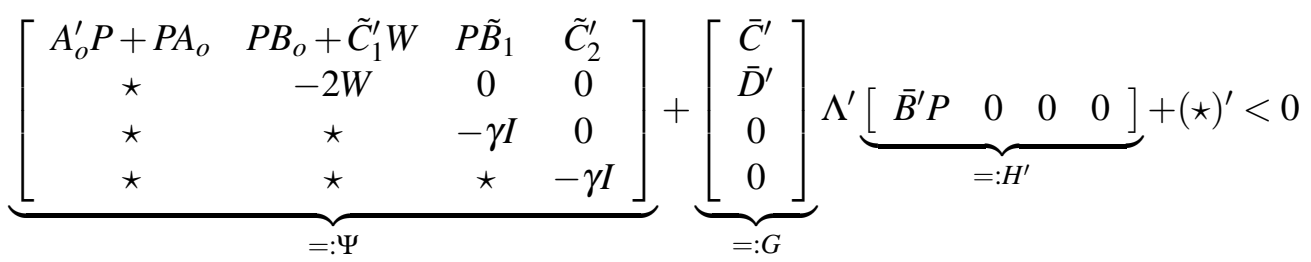

Using Lemma 1, this holds iff

$$
G^{\perp} \Psi\left(G^{\perp}\right)^{\prime}<0 \quad \text { and } \quad H^{\perp} \Psi\left(H^{\perp}\right)^{\prime}<0
$$

The remainder of the proof evaluates these two expressions for $G$ and $H$ defined above.

(i) $G^{\perp} \Psi\left(G^{\perp}\right)^{\prime}$. Note that $G \in \mathbb{R}^{\left(2 n_{p}+n_{a w}+n_{c}+q+n_{w}+n_{z}\right) \times\left(n_{a w}+q\right)}$ can be written as

$$
G=\left[\begin{array}{cc}
0 & 0 \\
0 & 0 \\
I & 0 \\
0 & C_{p}^{\prime} \\
\hline 0 & I \\
\hline 0 & 0 \\
\hline 0 & 0
\end{array}\right] \quad \text { with } \operatorname{rank}(G)=n_{a w}+q
$$


Thus the null-space of $G^{\prime}$ has dimension $2 n_{p}+n_{c}+n_{w}+n_{z}$ and a matrix of maximal rank whose columns span this null-space can be constructed as

$$
G^{\perp}=\left[\begin{array}{cccc|c|c|c}
I & 0 & 0 & 0 & 0 & 0 & 0 \\
0 & I & 0 & 0 & 0 & 0 & 0 \\
0 & 0 & 0 & I & -C_{p}^{\prime} & 0 & 0 \\
\hline 0 & 0 & 0 & 0 & 0 & I & 0 \\
\hline 0 & 0 & 0 & 0 & 0 & 0 & I
\end{array}\right]=:\left[\begin{array}{c|c|c|c}
I_{1}^{\prime} & I_{2}^{\prime} & 0 & 0 \\
\hline 0 & 0 & I & 0 \\
\hline 0 & 0 & 0 & I
\end{array}\right]
$$

Thus it follows that $G^{\perp} \Psi\left(G^{\perp}\right)^{\prime}<0$ is equivalent to

$$
\left[\begin{array}{ccc}
I_{1}^{\prime}\left(A_{o}^{\prime} P+P A_{o}\right) I_{1}+I_{1}^{\prime}\left(P B_{o}+\tilde{C}_{1}^{\prime} W\right) I_{2}+I_{2}^{\prime}\left(B_{o}^{\prime} P+W \tilde{C}_{1}\right) I_{1}-2 I_{2}^{\prime} W I_{2} & I_{1}^{\prime} P \tilde{B}_{1} & I_{1}^{\prime} \tilde{C}_{2}^{\prime} \\
\star & -\gamma I & 0 \\
\star & \star & -\gamma I
\end{array}\right]<0
$$

If, without loss of generality ${ }^{\dagger}, P$ is structured as

$$
P=\left[\begin{array}{cccc}
P_{11} & P_{12} & P_{13} & 0 \\
\star & P_{22} & P_{23} & 0 \\
\star & \star & P_{33} & 0 \\
\star & \star & \star & P_{44}
\end{array}\right]>0
$$

then extensive algebra proves that inequality (41) can be written as

$$
\left[\begin{array}{cccc}
A_{\text {nom }}^{\prime} P_{\text {nom }}+P_{\text {nom }} A_{\text {nom }} & P_{\text {nom }} B_{0, \text { nom }}+C_{0, \text { nom }}^{\prime}(W) & P_{\text {nom }} B_{1, \text { nom }} & C_{2, \text { nom }}^{\prime} \\
\star & A_{p}^{\prime} P_{44}+P_{44} A_{p}-2 C_{p}^{\prime} W C_{p} & P_{44}\left[0-B_{p d}\right] & 0 \\
\star & \star & -\gamma I & 0 \\
\star & \star & \star & -\gamma I
\end{array}\right]<0
$$

Applying the congruence transformation blockdiag $\left(P_{\text {nom }}^{-1}, I, I, I\right)$, and defining $R_{\text {nom }}:=P_{\text {nom }}^{-1}$, then gives inequality (32) in the theorem.

(ii) $H^{\perp} \Psi\left(H^{\perp}\right)^{\prime}$. Note that $H$ can be written as

$$
H=\operatorname{blockdiag}(P, I, I, I) \underbrace{\left[\begin{array}{cccc}
\bar{B}^{\prime} & 0 & 0 & 0
\end{array}\right]^{\prime}}_{\tilde{H}}
$$

Thus $H^{\perp}=\tilde{H}^{\perp} \operatorname{blockdiag}\left(P^{-1}, I, I, I\right)$, where $\tilde{H} \in \mathbb{R}^{\left(2 n_{p}+n_{a w}+n_{c}+q+n_{w}+n_{z}\right) \times\left(n_{a w}+n_{c}+m\right)}$ is given by

$$
\tilde{H}=\left[\begin{array}{ccc}
0 & 0 & B_{p} \\
0 & I & 0 \\
I & 0 & 0 \\
0 & 0 & 0 \\
\hline 0 & 0 & 0 \\
\hline 0 & 0 & 0 \\
\hline 0 & 0 & 0
\end{array}\right] \quad \text { with } \operatorname{rank}(\tilde{H})=n_{a w}+n_{c}+m
$$

The null space of $\tilde{H}$ has dimension $2 n_{p}-m+q+n_{w}+n_{z}$. Thus we can calculate $\tilde{H}^{\perp}$ as

$$
\tilde{H}^{\perp}=\left[\begin{array}{cccc|c|c|c}
B_{p}^{\perp} & 0 & 0 & 0 & 0 & 0 & 0 \\
0 & 0 & 0 & I & 0 & 0 & 0 \\
\hline 0 & 0 & 0 & 0 & I & 0 & 0 \\
\hline 0 & 0 & 0 & 0 & 0 & I & 0 \\
\hline 0 & 0 & 0 & 0 & 0 & 0 & I
\end{array}\right]=:\left[\begin{array}{c|c|c|c}
J_{1}^{\prime} & 0 & 0 & 0 \\
\hline 0 & I & 0 & 0 \\
\hline 0 & 0 & I & 0 \\
\hline 0 & 0 & 0 & I
\end{array}\right]
$$

\footnotetext{
${ }^{\dagger}$ Due to the triangular structure of $\tilde{A}$, there is no loss in generality of enforcing $P$ to be block-diagonal - see appendix for more details
} 
Thus letting $Q:=P^{-1}$ we get

$$
H^{\perp} \Psi\left(H^{\prime}\right)^{\perp}=\left[\begin{array}{cccc}
J_{1}^{\prime}\left(Q A_{o}^{\prime}+A_{o} Q\right) J_{1} & J_{1}^{\prime}\left(B_{o}+Q \tilde{C}_{1}^{\prime} W\right) & J_{1}^{\prime} \tilde{B}_{1} & J_{1}^{\prime} Q \tilde{C}_{2}^{\prime} \\
\star & -2 W & 0 & 0 \\
\star & \star & -\gamma I & 0 \\
\star & \star & \star & -\gamma I
\end{array}\right]<0
$$

Using the structure of $P$ given in equation (42), $Q=P^{-1}$ is defined as

$$
Q:=\left[\begin{array}{cccc}
Q_{11} & Q_{12} & Q_{13} & 0 \\
\star & Q_{22} & Q_{23} & 0 \\
\star & \star & Q_{33} & 0 \\
\star & \star & \star & Q_{44}
\end{array}\right]
$$

Labourious algebra then shows that inequality (47) is equivalent to

$$
\left[\begin{array}{ccccc}
B_{p}^{\perp}\left(Q_{11} A_{p}^{\prime}+A_{p} Q_{11}\right)\left(B_{p}^{\perp}\right)^{\prime} & 0 & B_{p}^{\perp} Q_{11} C_{p}^{\prime} W & B_{p}^{\perp} B_{p d} & B_{p}^{\perp} Q_{11} C_{p z}^{\prime} \\
\star & Q_{44}\left(A_{p}+L C_{p}\right)^{\prime}+\left(A_{p}+L C_{p}\right) Q_{44} & L & -B_{p d} & 0 \\
\star & \star & -2 W & 0 & 0 \\
\star & \star & \star & -\gamma I & 0 \\
\star & \star & \star & \star & -\gamma I
\end{array}\right]<0
$$

The congruence transformation blockdiag $\left(I, Q_{44}^{-1}, I, I, I\right)$ can then be used to arrive at inequality (31) in the theorem.

iii) Ensuring $P>0$

Quadratic stability holds under the condition that $P>0$, which is equivalent to inequality (42) holding. In turn this will hold if $P_{44}>0, Q_{n o m}>0$ and there exist matrices, $\left[\begin{array}{lll}Q_{13}^{\prime} & Q_{23}^{\prime}\end{array}\right]^{\prime}, \quad\left[\begin{array}{ll}P_{13}^{\prime} & P_{23}^{\prime}\end{array}\right]^{\prime} \in \mathbb{R}^{\left(n_{p}+n_{c}\right) \times n_{a w}}$ and $P_{33}, Q_{33} \in \mathbb{R}^{n_{a w} \times n_{a w}}$ such that the following inequality holds

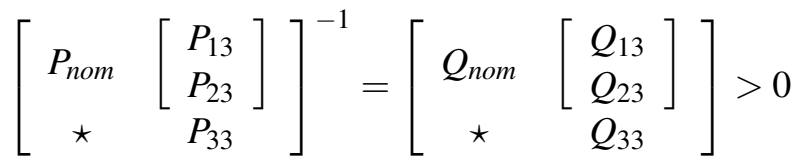

Now, from Lemma 3 these matrices exist if and only if the following inequalities hold

$$
\begin{aligned}
Q_{\text {nom }}-P_{\text {nom }}^{-1} & \geq 0 \\
\operatorname{rank}\left(Q_{\text {nom }}-P_{n o m}^{-1}\right) & \leq n_{a w}
\end{aligned}
$$

Recalling that $P_{\text {nom }}^{-1}=R_{\text {nom }}$ yields inequalities (33) and (34). This completes the proof.

The existence conditions in Theorem 1 are "almost" linear matrix inequalities in the variables $Q_{\text {nom }}, R_{\text {nom }}, W$ and $\gamma$ with two sources of nonlinearity preventing them being linear. The first is the obvious non-convex rankconstraint in inequality (34) which often occurs in LMI controller design [5]. However, as in [8], for certain orders of anti-windup compensator, particularly $n_{a w} \geq n_{p}$ and $n_{a w}=0$, the rank constraint will vanish. The second source of nonlinearity is the bilinear term involving $Q_{11}$ and $W$, although for fixed $W$ this becomes linear and hence the inequalities become linear. Unlike the corresponding conditions for the input saturation case ([8]) note that the "multiplier" matrix $W$ is still present in the existence conditions.

\section{Discussion}

When the anti-windup compensator is chosen to be of order $n_{p}$ or zero, Theorem 1 gives matrix inequalities which can be checked for existence. However, they do not reveal any intrinsic properties about the class of 
systems for which they are satisfied, nor do they reveal lower bound on the $\mathscr{L}_{2}$ gain. A lower bound on the $\mathscr{L}_{2}$ gain can easily checked by omitting the 2 nd and 3 rd rows and columns of inequality (31) and the second column of inequality (32) to yield.

$$
\begin{gathered}
{\left[\begin{array}{ccc}
B_{p}^{\perp}\left(Q_{11} A_{p}^{\prime}+A_{p} Q_{11}\right)\left(B_{p}^{\perp}\right)^{\prime} & B_{p}^{\perp} B_{p d} & B_{p}^{\perp} Q_{11} C_{p z}^{\prime} \\
\star & -\gamma I & 0 \\
\star & \star & -\gamma I
\end{array}\right]<0} \\
{\left[\begin{array}{ccc}
R_{\text {nom }} A_{\text {nom }}^{\prime}+A_{\text {nom }} R_{\text {nom }} & B_{1, \text { nom }} & R_{\text {nom }} C_{2, \text { nom }}^{\prime} \\
\star & -\gamma I & 0 \\
\star & \star & -\gamma I
\end{array}\right]<0}
\end{gathered}
$$

These two equations are in the form of "bounded real lemma" inequalities, which imply that the $\mathscr{L}_{2}$ gain is lower bounded by the $\mathscr{H}_{\infty}$ norm of the closed-loop system, $\left\|[G, K]_{\text {nom }}\right\|_{\infty}$, and the $\mathscr{H}_{\infty}$ norm of a system very closely related to the open-loop plant (as given in inequality (53)). This is a similar observation to that made in the case of anti-windup for systems with input saturation in [8], although added complications with the structure of inequalities (31) and (32), make the bound above more conservative (due to the extra constraints in the 2nd and 3 rd rows) than in the case of anti-windup compensation for systems with input saturation.

\subsection{Simplified existence conditions}

It is interesting to examine the properties of systems for which there exists an anti-windup compensator guaranteeing global quadratic stability and $\mathscr{L}_{2}$ gain. Although conditions are given in Theorem 1 , it is desirable to reveal deeper properties which the nominal linear system must possess in order for these conditions to be satisfied. The main result of this section is

Corollary 1 Assume $L=0$, then an anti-windup compensator $\Lambda(s)$ which satisfied the conditions of Theorem 1 exists if and only if

\section{1. $A_{p}$ is Hurwitz}

2. $A_{\text {nom }}$ is Hurwitz

Proof: Theorem 1 will be satisfied for sufficiently large $\gamma$ providing that the following conditions hold:

$$
\begin{aligned}
& {\left[\begin{array}{ccc}
B_{p}^{\perp}\left(Q_{11} A_{p}^{\prime}+A_{p} Q_{11}\right)\left(B_{p}^{\perp}\right)^{\prime} & 0 & B_{p}^{\perp} Q_{11} C_{p}^{\prime} W \\
\star & \left(A_{p}+L C_{p}\right)^{\prime} P_{44}+P_{44}\left(A_{p}+L C_{p}\right) & P_{44} L \\
\star & \star & -2 W
\end{array}\right]<0} \\
& {\left[\begin{array}{cc}
R_{\text {nom }} A_{\text {nom }}^{\prime}+A_{\text {nom }} R_{\text {nom }} & B_{0, \text { nom }}+R_{\text {nom }} C_{0, \text { nom }}^{\prime}(W) \\
\star & A_{p}^{\prime} P_{44}+P_{44} A_{p}-2 C_{p}^{\prime} W C_{p}
\end{array}\right]<0} \\
& Q_{\text {nom }}-R_{\text {nom }} \geq 0 \\
& \operatorname{rank}\left(Q_{\text {nom }}-R_{\text {nom }}\right) \leq n_{\text {aw }}
\end{aligned}
$$

We shall examine these conditions in turn.

Inequality (56). Letting $L=0$ and applying the Schur complement, it follows that inequality (56) is equivalent to.

$$
\begin{aligned}
A_{p}^{\prime} P_{44}+P_{44} A_{p} & <0 \\
B_{p}^{\perp}\left(Q_{11} A_{p}^{\prime}+A_{p} Q_{11}+Q_{11} C_{p}^{\prime} W C_{p} Q_{11}\right)\left(B_{p}^{\perp}\right)^{\prime} & <0
\end{aligned}
$$


These conditions will hold iff if $A_{p}$ is Hurwitz.

Inequality (57). Necessity. From the $(1,1)$ element it is trivial that $A_{\text {nom }}$ must be Hurwitz. To see that $A_{p}$ also must be Hurwitz, we apply the congruence transformation

$$
\left[\begin{array}{cc}
R_{n o m}^{-1} & {\left[\begin{array}{c}
-I \\
0 \\
0
\end{array}\right]}
\end{array}\right]
$$

to obtain the equivalent inequality

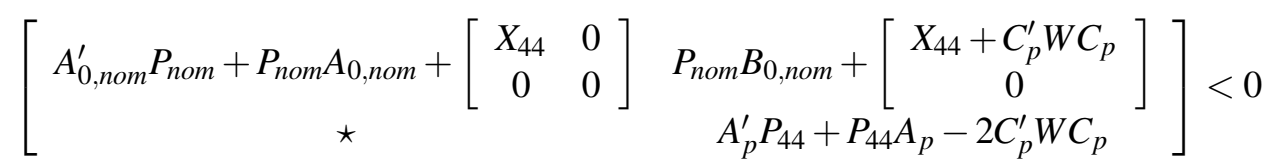

where $X_{44}=A_{p}^{\prime} P_{44}+P_{44} A_{p}$. Simple algebra shows that the $(1,1)$ element is negative definite if and only if $A_{p}$ is Hurwitz and $\left(B_{p} C_{c}, A_{c}\right)$ is detectable, which is implied by $A_{\text {nom }}$ being Hurwitz.

Sufficiency. Assume, $A_{n o m}$ and $A_{p}$ are Hurwitz, then, choosing $P_{44}$ sufficiently large ensures that inequality (57) holds.

Conditions (58) and (59). Assume that $n_{a w}=n_{p}$, then condition (59) is trivially satisfied. Next let,

$$
Q_{n o m}=\left[\begin{array}{cc}
Q_{11} & R_{12} \\
\star & R_{22}
\end{array}\right]
$$

Then choosing $Q_{11}$ sufficiently large ensures that inequality (58) is satisfied.

Remark 4 Again, it is useful to constrast these results with those in [8] which correspond to the case of actuator rather than sensor saturation. The fundamental existence conditions are effectively the same in both cases: global exponential stability of both the plant and the un-saturated closed-loop is a requirement for there to exist an antiwindup compensator ensuring global quadratic stability and finite $\mathscr{L}_{2}$ gain. These conditions were also hinted at in [19]. However, the $\mathscr{L}_{2}$ gain bound is somewhat different in the sensor saturation case because (i) the presence of the observer adds extra constraints to this bound (see inequalities (31) and (32)) and (ii), the "bounded real" inequality for the open-loop (which contributes to a lower bound on the $\mathscr{L}_{2}$ gain) is modified by "outer factors" which depend on $B_{p}^{\perp}$. In particular the dependence of the $\mathscr{L}_{2}$ gain bound on $B_{p}^{\perp}$ implies that the more actuated a system is, the fewer constraints on the $\mathscr{L}_{2}$ gain there will be.

\section{Example}

To illustrate the use of anti-windup compensation for sensor saturation, Theorem 1 is applied to a simple example. Consider the plant

$$
\left[\begin{array}{c||c|c}
A_{p} & B_{p} & B_{p d} \\
\hline \hline C_{p} & 0 & 0 \\
\hline C_{p z} & 0 & 0
\end{array}\right]=\left[\begin{array}{ccc||c|c}
-2.2000 & -0.2050 & -0.0100 & 1 & 0.001 \\
2.0000 & 0 & 0 & 0 & 0 \\
0 & 1.0000 & 0 & 0 & 0 \\
\hline 1.0000 & 0.4000 & 0.0750 & 0 & 0 \\
\hline 1.0000 & 0.4000 & 0.0750 & 0 & 0
\end{array}\right]
$$

and the linear controller

$$
\left[\begin{array}{c||c|c}
A_{c} & B_{c} & B_{c r} \\
\hline \hline C_{c} & D_{c} & D_{c r}
\end{array}\right]=\left[\begin{array}{c||c|c}
0 & -4 & 4 \\
\hline \hline 4.25 & -17 & 17
\end{array}\right]
$$

The closed-loop interconnection $[G, K]_{\text {nom }}$ of these two elements is asymptotically stable; the plant is also asymptotically stable. Thus from Corollary 1 , we know that an anti-windup compensator enforcing global exponential 
stability and $\mathscr{L}_{2}$ gain will exist. An observer for this system is constructed according equation (10) with $L=0$ (i.e. the observer is just a copy of the plant, as proposed in [19]).

An anti-windup compensator was designed using Theorem 1 , with $W=1$. Using standard controller re-construction algorithms (given in [8] and [5] for example), the following anti-windup compensator was obtained

$$
\left[\begin{array}{c|c}
\Lambda_{1} & \Lambda_{2} \\
\hline \hline \Lambda_{31} & \Lambda_{32} \\
\hline \Lambda_{41} & \Lambda_{42}
\end{array}\right]=\left[\begin{array}{ccc|c}
-308.3860 & -18.9999 & -12.8327 & -21.1642 \\
-37.2891 & -4.1645 & -0.9023 & -1.5614 \\
-1.3277 & 1.4301 & -1.5368 & -0.6332 \\
\hline \hline-12.7091 & -1.8722 & 0.0113 & -8.1895 \\
\hline-6679.9 & -424.6887 & -272.5045 & -487.7512
\end{array}\right]
$$

which corresponded to an $\mathscr{L}_{2}$ gain of $\gamma=1.0254$.

Figure 8 shows the system's response, $y(t)$ to a pulse input of magnitude 2 units in various circumstances. The dotted line shows the response when no sensor saturation is present, and thus the anti-windup compensator is not active. The dashed line shows the response when sensor saturation with limits $\bar{y}=1.5$ units is introduced. Notice the sharp degradation in performance and the excessive overshoot in the response. The solid line shows the response of the system with the same level of sensor saturation but with the above anti-windup compesator active. Although the system cannot recover the precise pulse characteristics, the deviation from the linear behaviour is substantially lower and the recovery of the linear response is much faster. Figure 9 shows the behaviour of the signal which is passed to the sensors (i.e. after saturation), $y_{m}(t)$. Observe that when anti-windup compensation is active, the sensor remains saturated for a shorter period of time and linear behaviour is resumed earlier.

Remark 5: Note that the results in [26] are more restrictive because that uses Architecture 1 which involves two saturation nonlinearities and is hence more conservative. Crucially, the results of [26] also require the linear controller to be exponentially stable which is not the case with the PI controller used here.

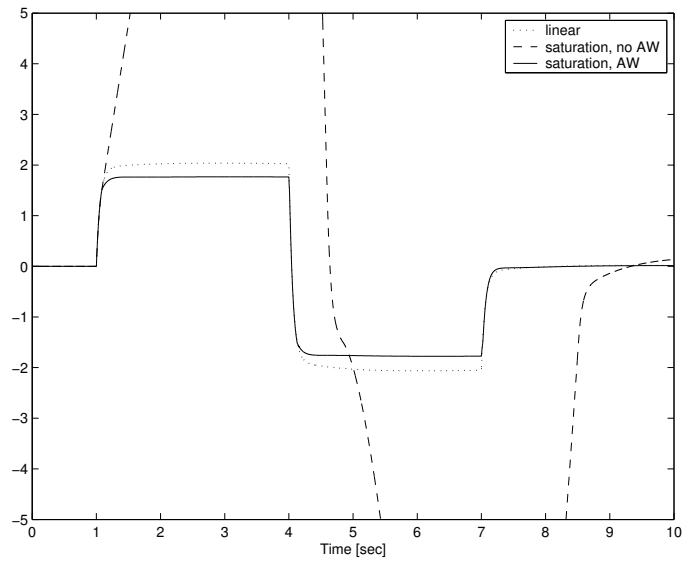

Figure 8: Output response $y(t)$ of example system

\section{Conclusion}

Necessary and sufficient conditions for the existence of an anti-windup compensator which guarantees quadratic stability and $\mathscr{L}_{2}$ gain of a linear system subject to a sensor saturation nonlinearity have been presented. An important corollary to these conditions implies that such an anti-windup compensator only exists if and only if the nominal open-loop and closed-loop linear systems are asymptotically stable. This implies that anti-windup compensators for systems with sensor saturation exist under the same conditions as they do for systems with input saturation; a fact which was first hinted at in [19], although no proof was supplied. However, due to the problem's 


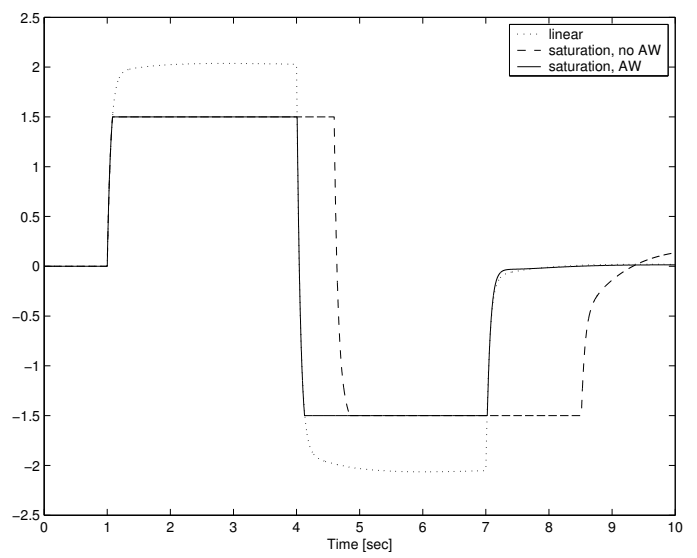

Figure 9: Sensor response $y_{m}(t)$ of example system

structure, the $\mathscr{L}_{2}$ gain bound for systems with sensor saturation is generally different to that for systems with input saturation. An example has presented the effectiveness of the proposed technique. As indicated in the introduction, sensor saturation could be used to approximate the case in which only the sign of the output is known and it would be of interest to investigate the use of the methods proposed here in such situations.

\section{References}

[1] S. Boyd, L. El Ghaoui, E. Feron, and V. Balakrishnan. Linear Matrix Inequalities in System and Control Theory. SIAM, 1994.

[2] Y.Y. Cao, Z. Lin, and B.M. Chen. An output feedback $\mathscr{H}_{\infty}$ controller for linear systems subject to sensor nonlinearities. IEEE Transactions on Circuits and Systems-I, 50(7):914-921, 2003.

[3] S. Crawshaw. Global and local analyses of coprime-factor based anti-windup for stable and unstable plants. In Proc. European Control Conference, Cambridge, 2003.

[4] A.T. Fuller. In-the-large stability of relay and saturating control systems with linear controller. International Journal of Control, 10:457-480, 1969.

[5] P. Gahinet and P. Apkarian. A linear matrix inequality approach to $\mathscr{H}_{\infty}$ control. International Journal of Robust and Nonlinear Control, 4:421-448, 1994.

[6] A. H. Glattfelder, W. Schaufelberger, and H.P. Fassler. Stability of override control systems. International Journal of Control, 37(5):1023-1037, 1983.

[7] J.M. Gomes da Silva Jr. and S. Tarbouriech. Anti-windup design with guaranteed region of stability: an LMI-based approach. IEEE Transactions on Automatic Control, 50(1):106-111, 2005.

[8] G. Grimm, J. Hatfield, I. Postlethwaite, A.R. Teel, M.C. Turner, and L. Zaccarian. Anti-windup for stable linear systems with input saturation: an LMI based synthesis. IEEE Transactions on Automatic Control, 48(9):1509-1525, 2003.

[9] G. Herrmann, M.C. Turner, and I. Postlethwaite. Discrete-time and sampled-data anti-windup synthesis: stability and performance. International Journal of Systems Science, 37(10), 2006.

[10] G. Kaliora and A. Astolfi. Nonlinear control of feedforward systems with bounded signals. IEEE Transactions on Automatic Control, 49(11):1975-1990, 2004. 
[11] R.B. Koplon, M.L.J. Hautus, and E.D. Sontag. Observability of linear systems with saturated outputs. Linear Algebra and Applications, 205-206:909-936, 1994.

[12] M.V. Kothare, P.J. Campo, M. Morari, and C.N. Nett. A unified framework for the study of anti-windup designs. Automatica, 30(12):1869-1883, 1994.

[13] G. Kreisselmeier. Stabilisation of linear systems in the presence of output measurement saturation. Systems and Control Letters, 29:27-30, 1996.

[14] Z. Lin and T. Hu. Semi-global stabilisation of linear systems subject to output saturation. Systems and Control Letters, 43:211-217, 2001.

[15] Z. Lin and A. Saberi. Semi-global exponential stabilisation of linear systems subject to input saturation via linear feedbacks. Systems and Control Letters, 21:225-239, 1996.

[16] V.R. Marcopoli and S.M. Phillips. Analysis and synthesis tools for a class of actuator-limited multivariable control systems: a linear matrix inequality approach. International Journal of Robust and Nonlinear Control, 6(9-10):1045-1063, 1996.

[17] T. Nguyen and F. Jabbari. Output feedback controllers for disturbance attenuation with actuator amplitude and rate saturation. Automatica, 36(9):1339-1346, 2000.

[18] A. Packard. Gain scheduling via linear fractional transformations. Systems and Control Letters, 22(2):7992, 1994.

[19] J.-K. Park and H.Y. Youn. Dynamic anti-windup based control method for state-constrained systems. Automatica, 39:1915-1922, 2003.

[20] S. Solyom. A synthesis method for static anti-windup compensators. In Proc. European Control Conference, Cambridge, 2003.

[21] H.J. Sussmann, E.D. Sontag, and Y. Yang. A general result on the stabilisation of linear systems using bounded controls. IEEE Transactions on Automatic Control, 39:2411-2425, 1994.

[22] S. Tarbouriech and G. Garcia. Preliminary results about anti-windup strategy for systems subject to actuator and sensor saturations. In Proc. of the IFAC World Congress, Prague, 2005.

[23] S. Tarbouriech, G. Garcia, and A.H. Glattfelder, editors. Advanced Strategies in Control Systems with Input and Output Constraints, volume 346 of Lecture Notes in Control and Infomrmation Sciences. Springer, 2007.

[24] A.R. Teel and N. Kapoor. The $\mathscr{L}_{2}$ anti-windup problem: Its definition and solution. In Proc. European Control Conference, Brussels, 1997.

[25] M.C. Turner and I. Postlethwaite. A new perspective on static and low order anti-windup synthesis. International Journal of Control, 77(1):27-44, 2004.

[26] M.C. Turner and S. Tarbouriech. Anti-windup compensation for systems with sensor saturation: sufficient conditions for stability and $\mathscr{L}_{2}$ gain. In Proc. of the IEEE Conference on Decision and Control, San Diego, 2006.

[27] M.C. Turner and L. Zaccarian, editors. Special Issue on Anti-windup, volume 37 of International Journal of Systems Science, 2006.

[28] N. Wada and M. Saeki. Design of a static anti-windup compensator which guarantees robust stability. Transactions of the Institute of Systems, Control and Instrumentation Enigneers, 12(11):664-670, 1999. 
[29] M.J. Walsh and M.J. Hayes. A robust throughput rate control mechanism for an 802.15.4 wireless sensor network - an anti-windup approach. In Proc. of the American Control Conference, New York, 2007.

[30] F. Wu and M. Soto. Extended anti-windup control schemes for lti and lft systems with actuator saturations. International Journal of Robust and Nonlinear Control, 14(15):1255-1281, 2004.

\section{A State-space matrices}

Nominal linear system. Closed-loop linear system:

$\left[\begin{array}{c|c|c}A_{\text {nom }} & B_{0, \text { nom }} & B_{1, \text { nom }} \\ \hline C_{1, \text { nom }} & 0 & 0 \\ \hline C_{2, \text { nom }} & 0 & 0\end{array}\right]=\left[\begin{array}{cc|c|cc}A_{p}+B_{p} D_{c} C_{p} & B_{p} C_{c} & B_{p} D_{c} C_{p} & B_{p} D_{c r} & B_{p d} \\ B_{c} C_{p} & A_{c} & B_{c} C_{p} & B_{c r} & 0 \\ \hline C_{p} & 0 & 0 & 0 & 0 \\ \hline C_{p z} & 0 & 0 & 0 & 0\end{array}\right]$

Open-loop linear system "A" matrix:

$$
A_{0, \text { nom }}=\left[\begin{array}{cc}
A_{p} & B_{p} C_{c} \\
0 & A_{c}
\end{array}\right]
$$

Dummy C-matrix

$$
C_{0, \text { nom }}(W)=\left[\begin{array}{ll}
-C_{p} W C_{p}^{\prime} & 0
\end{array}\right]
$$

$\underline{\text { Saturated system }}$

$$
\begin{aligned}
\tilde{A} & =\left[\begin{array}{cccc}
A_{p}+B_{p} D_{c} C_{p} & B_{p} C_{c} & B_{p} \Lambda_{32} & B_{p} \Lambda_{42} C_{p} \\
B_{c} C_{p} & A_{c} & \Lambda_{31} & \Lambda_{41} C_{p} \\
0 & 0 & \Lambda_{1} & \Lambda_{2} C_{p} \\
0 & 0 & 0 & A_{p}+L C_{p}
\end{array}\right] \\
\tilde{B}_{1} & =\left[\begin{array}{cc}
B_{p} D_{c r} & B_{p d} \\
B_{c r} & 0 \\
0 & 0 \\
0 & -B_{p d}
\end{array}\right] \quad \tilde{B}_{2}=\left[\begin{array}{c}
B_{p}\left(\Lambda_{42}-D_{c}\right) \\
\Lambda_{41}-B_{c} \\
\Lambda_{2} \\
L
\end{array}\right] \\
\tilde{C}_{1} & =\left[\begin{array}{llll}
C_{p} & 0 & 0 & 0
\end{array}\right] \\
\tilde{C}_{2} & =\left[\begin{array}{llll}
C_{p z} & 0 & 0 & 0
\end{array}\right]
\end{aligned}
$$

\section{Partitioned saturated system}

$$
\begin{aligned}
A_{0}= & {\left[\begin{array}{cccc}
A_{p} & B_{p} C_{c} & 0 & 0 \\
B_{c} C_{p} & A_{c} & 0 & 0 \\
0 & 0 & 0 & 0 \\
0 & 0 & 0 & A_{p}+L C_{p}
\end{array}\right] \quad B_{0}=\left[\begin{array}{c}
-B_{p} D_{c} \\
-B_{c} \\
0 \\
L
\end{array}\right] } \\
\bar{B} & =\left[\begin{array}{ccc}
0 & 0 & B_{p} \\
0 & I & 0 \\
I & 0 & 0 \\
0 & 0 & 0
\end{array}\right] \quad \Lambda=\left[\begin{array}{cc}
\Lambda_{1} & \Lambda_{2} \\
\Lambda_{31} & \Lambda_{32} \\
\Lambda_{41} & \Lambda_{42}
\end{array}\right] \\
\bar{C} & =\left[\begin{array}{llll}
0 & 0 & I & 0 \\
0 & 0 & 0 & C_{p}
\end{array}\right] \quad \bar{D}=\left[\begin{array}{l}
0 \\
I
\end{array}\right]
\end{aligned}
$$




\section{B On the use of diagonal Lyapunov functions}

In this section we show that, without loss of generality when one is seeking a Lyapunov function to enforce the Circle Criterion for $\Psi(.) \in$ Sector $[0, I]$, then if the "A" matrix has a triangular structure, then without loss of generality one can assume a diagonal structure for the Lyapunov function. Formally this is stated as

\section{Lemma 4 Consider the inequality}

$$
\left[\begin{array}{cc}
A^{\prime} P+P A & P B+C^{\prime} W \\
\star & -2 W
\end{array}\right]<0
$$

where A is block triangular, viz

$$
A=\left[\begin{array}{c|c}
A_{11} & A_{12} \\
\hline 0 & A_{22}
\end{array}\right] \in \mathbb{R}^{\left(n_{1}+n_{2}\right) \times\left(n_{1}+n_{2}\right)} \quad B=\left[\frac{B_{11}}{B_{21}}\right] \in \mathbb{R}^{\left(n_{1}+n_{2}\right) \times m} \quad C=\left[C_{11} \mid C_{12}\right] \in \mathbb{R}^{m \times\left(n_{1}+n_{2}\right)}
$$

Then there exist matrices $P \in \mathbb{R}_{+}^{\left(n_{1}+n_{2}\right) \times\left(n_{1}+n_{2}\right)}$ and $W \in \mathbb{D}_{+}^{m \times m}$ which satisfies this inequality if and only if there exists matrices $P=\operatorname{blockdiag}\left(P_{11}, P_{22}\right) \in \mathbb{R}_{+}^{\left(n_{1}+n_{2}\right) \times\left(n_{1}+n_{2}\right)}$ and $W \in \mathbb{D}_{+}^{m \times m}$ which also satisfy the above inequality.

Proof: The proof is a simple application of Finsler's lemma. Note that in terms of the matrices $A, B, C$, the above inequality can be written as

$$
\underbrace{\left[\begin{array}{ccc}
A_{11}^{\prime} P_{11}+P_{11} A_{11} & P_{11} A_{12} & P_{11} B_{11}+C_{11}^{\prime} W \\
\star & A_{22}^{\prime} P_{22}+P_{22} A_{22} & P_{22} B_{21}+C_{12}^{\prime} W \\
\star & -2 W
\end{array}\right]}_{\Psi_{M}}+\left[\begin{array}{cc}
I & A_{11}^{\prime} \\
0 & A_{12}^{\prime} \\
0 & B_{11}^{\prime}
\end{array}\right] P_{12}\left[\begin{array}{ccc}
0 & A_{22} & B_{21} \\
0 & I & 0
\end{array}\right]+(\star)^{\prime}<0
$$

Using Finsler's Lemma and the Schur complement, it follows that inequality (76) is satisfied if and only if $\exists \sigma \in \mathbb{R}$ such that

$$
\begin{aligned}
& {\left[\begin{array}{ccccc}
A_{11}^{\prime} P_{11}+P_{11} A_{11} & P_{11} A_{12} & P_{11} B_{11}+C_{11}^{\prime} W & I & A_{11}^{\prime} \\
\star & A_{22}^{\prime} P_{22}+P_{22} A_{22} & P_{22} B_{21}+C_{12}^{\prime} W & 0 & A_{12}^{\prime} \\
\star & \star & -2 W & 0 & B_{11}^{\prime} \\
\star & \star & \star & -\sigma^{-1} I & 0 \\
\star & \star & \star & \star & -\sigma^{-1} I
\end{array}\right]<0} \\
& {\left[\begin{array}{ccccc}
A_{11}^{\prime} P_{11}+P_{11} A_{11} & P_{11} A_{12} & P_{11} B_{11}+C_{11}^{\prime} W & 0 & 0 \\
\star & A_{22}^{\prime} P_{22}+P_{22} A_{22} & P_{22} B_{21}+C_{12}^{\prime} W & A_{22}^{\prime} & I \\
\star & \star & -2 W & B_{21}^{\prime} & 0 \\
\star & \star & \star & -\sigma^{-1} I & 0 \\
\star & \star & \star & \star & -\sigma^{-1} I
\end{array}\right]<0}
\end{aligned}
$$

Necessity. For both inequalities (77) and (78) to hold, it is necesssary that $\Psi_{M}<0$.

Sufficiency. If $\Psi_{M}<0$, then it follows that there always exists a sufficiently small $\sigma$ such that both inequalities (77) and (78) hold.

Hence $\Psi_{M}<0$ is both necessary and sufficient for the inequality (74) to hold, providing the matrices are structured as above. Notice that $\Psi_{M}<0$ is nothing more than inequality (74) with $P=\operatorname{blockdiag}\left(P_{11}, P_{22}\right)$. This completes the proof. 